${ }^{10}$ C. C. Chang, J. R. Wu, T. W. Koontz, and H. D. Holmgren, Bull. Am. Phys. Soc. 23, 929 (1978).

${ }^{11}$ S. Ferroni, G. Ricco, G. A. Rottigini, M. Sanzone, and G. LoBianco, Nuovo Cimento $\underline{35}, 15$ (1976).

${ }^{12}$ F. Ajzenberg-Selove, Nucl. Phys。 A268, 1 (1976), and references contained therein.

\title{
Bender-Wu Formula and the Stark Effect in Hydrogen
}

\author{
L. Benassi and V. Grecchi \\ Istituto Matematico and Istituto di Fisica, Università di Modena, Modena, Italy
}

and

E. Harrell

Department of Mathematics, Massachusetts Institute of Technology, Cambridge, Massachusetts 02139

and

B. Simon

Department of Mathematics and Department of Physics, Princeton University, Princeton, New Jersey 08540 (Received 23 October 1978)

\begin{abstract}
We discuss a close connection between the formula of Banks, Bender, and Wu for the asymptotics of the Rayleigh-Schrödinger coefficients of the two-dimensional rotationally symmetric anharmonic oscillator and the behavior of resonances of the hydrogen Stark problem in two regimes: small field (Oppenheimer's formula) and large field (where we obtain the new results $\arg E \rightarrow-\pi / 3,|E| \sim \alpha[F(\ln F)]^{2 / 3}$ for $F$, the electric field strength, going to infinity). We also announce a rigorous proof of Bender-Wu-type formulas.
\end{abstract}

In this note, we wish to discuss various aspects of the hydrogen Stark Hamiltonian (in atomic units)

$$
H=-\frac{1}{2} \Delta-Z r^{-1}+F x_{3},
$$

and, in particular, the celebrated formula of Oppenheimer ${ }^{1}$ for the width, $\Gamma$, of the ground state in small positive field $(Z=1)$,

$$
\Gamma=-2 \operatorname{Im} E(F)=4 F^{-1} \exp \left(-\frac{2}{3} F^{-1}\right)[1+O(F)] .
$$

We will link this closely to certain ideas developed by Bender and $\mathrm{Wu}$ during the past ten years ${ }^{2}$ involving the large- $n$ behavior of the RayleighSchrödinger coefficients of the ground state of an anharmonic oscillator. Equation (1) is connected ${ }^{3}$ to the Hamiltonian

$$
h=-d^{2} / d x^{2}+\left(m^{2}-\frac{1}{4}\right) x^{-2}+\alpha x^{2}+\beta x^{4}
$$

with the boundary conditions ${ }^{4}$

$$
u(0)=0(m=1,2, \ldots), u(x) \sim x^{1 / 2}(m=0),
$$

which is the Hamiltonian of the two-dimensional oscillator $-\Delta+\alpha r^{2}+\beta r^{4}$ reduced to the subspace $\left\{f(r) e^{\operatorname{Im} \Phi}\right\}$. We will let $\mu_{n}{ }^{(m)}(\alpha, \beta)$ denote the $(n$ +1 )th eigenvalue of (3). Using the Bender-Wu methods, $h$ was studied by Banks, Bender, and $\mathrm{Wu},{ }^{5}$ who found, in particular, that the ground- state Rayleigh-Schrödinger coefficients $a_{n}$, defined by

$$
u_{0}^{(0)}(1, \beta) \sim \sum a_{n} \beta^{n}
$$

obey $(n \sim \infty)$

$$
a_{n} \sim(8 / \pi)\left(\frac{3}{2}\right)^{n+1}(-1)^{n} n !\left[1+O\left(n^{-1}\right)\right] .
$$

Here we will discuss three results whose technical details will appear elsewhere ${ }^{6,7}$ :

(A) The equivalence of Eqs. (2) and (6) modulo technical conditions ${ }^{6,7}$;

(B) a rigorous proof of formulas of Bender-Wutype including the one-dimensional anharmonic oscillator, formula (6) and formula (2) (Ref. 6);

(C) an analysis of the relation between (6) and the large $-F$ behavior of $E(F)$ (Ref. 7); in particular, for one state that we consider here (which is probably ${ }^{8}$ the continuation of the ground state) for $F \sim \infty$,

$$
\begin{aligned}
& \arg E \sim-\pi / 3+O\left((\ln F)^{-1}\right), \\
& \begin{aligned}
|E| \sim \alpha F^{2 / 3}(\ln F)^{2 / 3}+\beta F^{2 / 3}(\ln \ln F)^{-1 / 3} \\
\\
\quad+O\left(F^{2 / 3}(\ln F)^{-1 / 3}\right),
\end{aligned}
\end{aligned}
$$


where ${ }^{9}$

$$
\begin{aligned}
& \alpha=2^{-5 / 3}=0.31498, \\
& \beta=\frac{8}{3} \alpha=0.83995 .
\end{aligned}
$$

As a preliminary to our discussion of these

$$
\operatorname{Im} \mu_{0}^{(0)}\left(1, e^{i \pi} \beta\right) \underset{\beta \downarrow 0}{\sim} 8 \beta^{-1} \exp \left(-\frac{2}{3} \beta^{-1}\right)[1+O(\beta)],
$$

and the result of $\operatorname{Simon}^{13}(n \geqslant 2)$,

$$
a_{n}=(-1)^{n} \pi^{-1} \int_{0}^{\infty} x^{-n-1} \operatorname{Im} \mu_{0}\left(1, e^{i \pi} x\right) d x .
$$

Roughly speaking,$^{14}(9)$ is equivalent to (6). We remark that the relation between (6) and (9) can be played also with $E$ so that by a result of Herbst and Simon, ${ }^{15}$ Eq. (2) implies that $E(F) \sim \sum A_{n} F^{2 n}$ with

$$
A_{n} \sim-4 \pi^{-1}\left(\frac{3}{2}\right)^{2 n+1}(2 n) ! .
$$

As a final preliminary, we recall the Symanzik scaling relation for $\mu_{n}$ (Ref. 13) which results from $x \rightarrow \lambda^{1 / 2} x$ :

$$
\mu_{n}\left(\lambda^{2} \alpha, \lambda^{3} \beta\right)=\lambda \mu_{n}(\alpha, \beta),
$$

and the resulting fact ${ }^{13}$ that if $\mu_{n}(1, \beta)$ is continued along circles $|\beta|=$ const, then 1

$$
\begin{aligned}
\mu_{n}\left(1, e^{6 \pi i} \beta\right) & =\mu_{n}(1, \beta) \\
& =-\mu_{n}\left(1, e^{3 \pi i} \beta\right)=\mu_{n}^{*}\left(1, \beta^{*}\right) .
\end{aligned}
$$

The key to the connection between (1) and (3) is the discovery of Titchmarsh ${ }^{16}$ that by use of squared parabolic coordinates, the equation $H \psi$ $=E \dot{ } \psi$ separates into two copies of (2) so that the points, we make several remarks. For $Z, F$ real, the Hamiltonian $H$ of Eq. (1) has a purely continuous spectrum ${ }^{10}$; resonance energies are defined by the method of complex scaling (dilation analyticity ${ }^{11}$ ) which has recently been extended to the hydrogen Stark problem. ${ }^{3,12}$ Secondly, Eq. (6) is actually deduced ${ }^{5}$ from the fact that

resonance eigenvalues $E(Z, F)$ are given by solutions of the implicit equation ${ }^{17}$

$$
\mu_{n_{1}}{ }^{(m)}(-2 E F)+\mu_{n_{2}}{ }^{(m)}\left(-2 E, F e^{-i \pi}\right)=4 Z .
$$

Using the scaling relation (11), (13) is equivalent to

$$
f(w)=\zeta,
$$

where

$$
f(w)=w^{-1}\left[\mu_{n_{1}}{ }^{(m)}\left(1, w^{3}\right)+\mu_{n_{2}}{ }^{(m)}\left(1, e^{-i \pi} w^{3}\right)\right],
$$

and

$$
w=F^{1 / 3} /(-2 E)^{1 / 2} ; \quad \zeta=4 Z / F^{1 / 3} .
$$

Noting that $w \zeta$ has a limit as $F \rightarrow 0$, we can identify the ground state ${ }^{18}$ of hydrogen $E(Z, 0)$ $=-\frac{1}{2} Z^{2}$ as $m=n_{1}=n_{2}=0$. Thus,

$$
\operatorname{Re}(w)=O\left(F^{1 / 3}\right),
$$

and therefore

$$
\begin{aligned}
\operatorname{Im}\left(w^{3}\right) & =O\left(\operatorname{Re}(w)^{2}\right) \operatorname{Im}(w)=O\left(F^{2 / 3}\right) \operatorname{Im}(w) \\
& =O(F) \operatorname{Im}(w \zeta),
\end{aligned}
$$

since $\zeta^{-1}=O\left(F^{1 / 3}\right)$. Multiplying Eq. (14) by $w$ and taking imaginary parts, we find that

$$
\operatorname{Im}(w \zeta)=\operatorname{Im} \mu_{0}{ }^{(0)}\left(1, e^{-i \pi} \operatorname{Re}\left(F /[-2 E(2, Z)]^{3 / 2}\right)\right)+O\left(\operatorname{Im}\left(w^{3}\right)\right),
$$

since $\mu_{0}{ }^{(0)}(1, \operatorname{Re}(--))$ is real and the derivatives of $\mu_{0}{ }^{(0)}$ with respect to $\beta$ are uniformly bounded as $\beta$ $\rightarrow 0,|\arg \beta|<\frac{3}{2} \pi-\delta .^{13}$ Thus, using (16) we see that

$$
\operatorname{Im}\left[42 /(-2 E)^{1 / 2}\right]=\operatorname{Im} \mu_{0}{ }^{(0)}\left(1, e^{-i \pi} F /[-2 E(Z, 0)]^{3 / 2}\right)[1+O(F)],
$$

so long as

$$
\operatorname{Im} \mu_{0}{ }^{(0)}\left(1, e^{-i \pi}\left[a F+O\left(F^{2}\right)\right]\right)=\operatorname{Im} \mu_{0}{ }^{(0)}\left(1, e^{i \pi} a F\right)[1+O(F)] .
$$

Thus, (2) is equivalent to (9).

As for point (B), we point out that in Ref. 6 a rigorous proof of (9) and also the original Bender $-\mathrm{Wu}^{2}$ formula is given. In this proof, a key role is played by a further transformation of Titchmarsh ${ }^{16}$ which relates (3) to the Schrödinger operator ${ }^{19}$ obtained by replacing $F x_{3}$ in (1) by $-F r$. A formula for the width is obtained in the model by a method whose first-order version is similar to the procedure of Bender and $\mathrm{Wu}^{2}$ of taking a trial wave function which is of WKB type in certain regions and "asymptotically exact ${ }^{20}$ " in others. We go further than they do by obtaining a convergent expansion ${ }^{21}$ allowing control on the possible errors. Sufficient control is obtained on derivatives to justify all formal ma- 
nipulations mentioned above and thus to prove (2). We note that our proof does not seem to shed any light on the recent functional integration demonstrations ${ }^{22,23}$ of the Bender-Wu formula.

Finally, we turn to a study of the limit $F \rightarrow+\infty, Z$ fixed. As a preliminary, we note that Herbst ${ }^{12}$ has shown that for $F$ fixed nonzero and $Z=0$, there are no resonances and no spectrum for the complex scaled $H$. It follows that for $F$ fixed, as $Z \rightarrow 0,|E| \rightarrow \infty$. Thus the only solutions of (14) with $\zeta \rightarrow 0$ have $w \rightarrow 0$ also. At first sight it does not appear that $f \rightarrow 0$ is possible as $w \rightarrow 0$ but if we change variables to $u=e^{i \pi / 3} w$, we see that

$$
f\left(e^{-i \pi / 3} u\right)=e^{i \pi / 3} u^{-1}\left[\mu_{n_{1}}\left(1, e^{-i \pi} u^{3}\right)+\mu_{n_{2}}\left(1, e^{-2 \pi i} u^{3}\right)\right]=e^{i \pi / 3} u^{-1}\left[\mu_{n_{1}}\left(1, e^{-i \pi} u^{3}\right)-\mu_{n_{2}}\left(1, e^{i \pi} u^{3}\right)\right],
$$

using the second equality in Eq. (12). Thus if $u \downarrow 0$ and $n_{1}=n_{2}, f$ will go to zero. More generally, the analysis ${ }^{13}$ of the asymptotics of $\mu_{n}$ shows that $f$ can only go to zero if $n_{1}=n_{2}$ and $u$ stays in the region $|\arg u| \leqslant \pi / 6$; thus as $F \rightarrow \infty$ along the real axis, $|E| \rightarrow \infty$ with

$$
-\frac{2}{3} \pi \leqslant \arg E \leqslant 0 \text {. }
$$

This agrees with the result ${ }^{24}$ that there are no resonances in a region $\left\{E\left|-\pi<\arg E<-\frac{2}{3} \pi-\epsilon ;\right| E \mid>M\right\}$ for $Z, F$ fixed. If we now suppose that the asymptotic formula (9), which can be written

$$
u^{-1} \mu_{0}\left(1, e^{-i \pi} u^{3}\right)-u^{-1} \mu_{0}\left(1, e^{i \pi} u^{3}\right) \sim-16 i u^{-4} \exp \left(-\frac{2}{3} u^{-3}\right)\left[1+O\left(u^{3}\right)\right],
$$

extends to $\mid$ argu| small, then (19) implies that (14) is equivalent to

$$
\exp \left(\frac{2}{3} w^{-3}\right)=\zeta w^{4}\left[C+O\left(w^{3}\right)\right]
$$

with $\pi / 6 \leqslant \arg w \leqslant \pi / 2$ and a nonzero constant $C$. Taking logarithms of (21) we find that

$$
w^{-3}=\frac{3}{2} \log \zeta+6 \ln w+O(1)=\frac{3}{2} \log \zeta-2 \ln \ln \zeta+O(1)=-\frac{1}{2} \log F-2 \ln \ln F+O(1)
$$

which implies (7) and (8).

We close by mentioning four important questions raised by part (C) of our analysis: (1) We have found a solution of (14) for each $m, n_{1}=n_{2}$ for $\zeta \sim 0$ ( $F$ large $)$ and $\zeta \sim \infty(F$ small $)$. If one takes the small $-F$ solution and tries to increase $F$, does it go over to the large- $F$ solution ${ }^{25}$ ?

(2) Is it possible to obtain (7),(8) without using the Titchmarsh reduction? This is relevant to the large- $F$ behavior of resonances in complex atoms. (3) The results of Herbst ${ }^{12}$ imply that for $Z, F$ fixed there are only finitely many resonances in any region of the $E$ plane. Since, for $F=0$, there are infinitely many bound states one expects infinitely many resonances $E_{n}(F)$, which by the above result of Herbst must have $\left|E_{n}(F)\right| \rightarrow \infty$ at $n \rightarrow \infty$. Since $w_{n}$ thus goes to zero while $f\left(w_{n}\right)$ stays finite, one would expect ${ }^{26}$ that $\arg u_{n} \rightarrow 0$ which would imply that $\arg E_{n}(F) \rightarrow-\pi / 3$ as $n \rightarrow \infty$. Is this true? (4) We expect that a resonance which solves (14) for $F$ small, $n_{1} \neq n_{2}$ will cross enough Bender-Wu cuts ${ }^{27}$ as $F$ is increased ${ }^{28}$ so that eventually $n_{1}=n_{2}$. Thus, for $F$ large, $n_{1}=n_{2}$, there would be many solutions of (14) all with $E$ having the same large $-F$ asymptotic behavior.

Can one prove this?

It is a pleasure to thank S. Graffi and I. Herbst for their interest and valuable comments. This research was supported in part by the National Science Foundation, Grant No. MCS 78-01885 and in part by the Istituto Nazionale di Fisica Nucleare, Sezione di Bologna. One of us (E.H.) acknowledges receipt of a National Science Foundation National Needs Fellowship.

${ }^{1}$ J. R. Oppenheimer, Phys. Rev. 31, 66 (1928); see also L. D. Landau and E. M. Lifshitz, Quantum Mechanics (Pergamon, New York, 1958).

${ }^{2} \mathrm{C}$. Bender and T. T.Wu, Phys. Rev。184, 1231 (1969), and Phys.Rev. Lett. 16, $461(1971)$, and Phys。 Rev. D 7, 1620 (1973).

${ }^{3}$ S. Graffi and V. Grecchi, Lett. Math. Phys。․, 335 (1978), and to be published.

${ }^{4}$ For $m=0$, all solutions of (3) go to zero, namely as $x^{1 / 2}$ or $x^{1 / 2} \ln x$, so we must specify boundary conditions carefully in that case.

${ }^{5}$ T. Banks, C. Bender, and T. T.Wu, Phys. Rev. D $\underline{8}, 3346$ (1973).

${ }^{6}$ E. Harrell and B. Simon, "The Mathematical Theory of Resonances Whose Widths are Exponentially Small" (to be published).

${ }^{7} \mathrm{~L}$. Benassi and V. Grecchi, "Resonances in the Stark Effect and Strongly Asymptotic Approximants" (to be published); this paper will also discuss numerical calculation of resonances.

${ }^{8}$ Of course we expect that the answer is yes; but we cannot rule out the bizarre possibility that as $F$ is in- 
creased from $0, E(F) \rightarrow \infty$ at some point $F_{1}$ and then at $F_{2}>F_{1}$ a new solution comes in from infinity which agrees with the $F \sim \infty$ as $F$ is increased.

${ }^{9}$ The exact values of $\alpha$ and $\beta$ are probably mainly of theoretical interest. For numerical purposes it is probably better to use the implicit Eq. (19); the situation reminds one of that in the large-field Zeeman effect; see J. Avron, I. Herbst, and B. Simon, (to be published).

${ }^{10} \mathrm{~J}$. Avron and I. Herbst, Commun. Math. Phys。52, 239 (1977); I. Herbst, Math. Zeit. 155, 55-70 (1977); B. Simon, to be published.

${ }^{11}$ An extensive review of both mathematical and computational aspects of complex scaling is to be published. See W. Reinhardt, Int. J. Quantum Chem. 10, 359 (1976), for calculations on the Stark problem.

${ }^{12}$ I. Herbst, to be published.

${ }^{13}$ B.Simon, Ann。 Phys。(N.Y.) 58, 76 (1970).

${ }^{14}$ (9) implies (6) but, rigorously speaking (6) only implies (9) in an averaged sense; but if one supposes that $\operatorname{Im} \partial \mu / \partial \beta$ and $\operatorname{Im} \mu$ have the same small- $\beta$ dependence, then (6) implies (9).

${ }^{15}$ I. Herbst and B. Simon, Phys. Rev. Lett. 41, 67 (1978)。

${ }^{16}$ E. C. Titchmarsh, Eigenfunction Expansions Associated with Second Order Differential Equations (Oxford Univ. Press, Oxford, 1958).

${ }^{17}$ The occurrence of $e^{-i \pi}$ rather than $e^{i \pi}$ in (13) is connected (Refs. 3 and 12) with the demand that $\operatorname{Im} E \leqslant 0$. With this choice, $E$ is an actual eigenvalue of $-\frac{1}{2} e^{-2 \theta}$ $\times \Delta-e^{-\theta} Z r^{-1}+e^{\theta} F x_{3}$ for $0<\operatorname{Im} \theta<\frac{1}{3} \pi$ as required by the general theory. The $m$ in (13) is the eigenvalue of $L_{Z}$ for the problem (1).

${ }^{18}$ It is merely for notational convenience that we consider $m=n_{1}=n_{2}=0$ 。 In Refs。 6 and 7 the general case is considered.

${ }^{19}$ This operator is further discussed in S. Graffi, V. Grecchi, S. Lavoni, and M. Maioli, (to be published). ${ }^{20}$ In the Bender-Wu case, the trial function is a para- bolic cylinder function in the near region; in our case, a Whittaker function-both are exact in the limit $\beta$, respectively $F$, goes to zero. Near the distant turning points Airy functions are used in both cases.

${ }^{21}$ The expansion is obtained by the method of variation of parameters from the theory of ordinary differential equations. This idea with first order being purely of WKB form appears already in N. Frömin and P. O. Frömin, JWKB Approximations, Contributions to the Theory (North- Holland, Amsterdam, 1965). Recently as expansion based on the asymptotic exact form in the non-WKB region has been introduced independently by W. Crutchfield, thesis, Princeton University (unpublished), and $\mathrm{E}$. Harrell, to be published. It is the latter idea that we use; see also E. Harrell, Commun. Math. Phys。 60,73 (1978).

${ }^{22}$ L. N. Lipatov, Pis'ma Zh. Eksp. Teor. Fiz. 25, 116 (1971) [JETP Lett. 25, 104 (1977)]; E. Brézin et al., Phys. Rev. D 15, 1544, 1558 (1977).

${ }^{23}$ One can go part way towards a rigorous justification of the path space argument, namely one can justify the method on $T^{-1} \operatorname{Tr}\{\exp [-T H(\beta)]\}$ for each fixed $T$. The situation is described in B. Simon, Functional Integration and Quantum Physics (Academic, New York, to be published).

${ }^{24} \mathrm{~S}$. Graffi and V. Grecchi, to be published.

${ }^{25}$ See Ref. 8.

${ }^{26}$ The only barrier to proving this is that we are dealing with $w_{n}{ }^{-1}\left[\mu_{n_{1}}\left(1, w_{n}^{3}\right)+\mu_{n_{2}}\left(1, w_{n}^{3} e^{i \pi}\right)\right]$ with $n_{1}, n_{2} \rightarrow \infty$ as $w_{n} \rightarrow 0$.

${ }^{27}$ In addition to the cubic "branch point" structure that $\mu(1, \beta)$ has at $\beta=0$, there are infinitely many singularities (believed to be square-root branch points) coalescing at $\beta=0, \arg \beta= \pm \frac{3}{2} \pi$, and our convention above is to draw circular arcs as branch cuts between these points. These are the Bender-Wu cuts. When one is crossed, two values of $n$ are interchanged.

${ }^{28} \mathrm{~A}$ calculation for $n_{1}=m=0, n_{2}=1$ suggests that this is precisely what happens; see Ref. 7 . 